\title{
Drug-Seeking Behavior Is Significantly Attenuated in Nutritionally Supplemented Cocaine Withdrawn Sprague-Dawley Rats
}

\author{
Lauriann Young ${ }^{*}{ }^{\mathbb{D}}$, Annice Webber-Waugh, Karen Thaxter \\ Physiology Section, Department of Basic Medical Sciences, The University of the West Indies (UWI), Mona, Jamaica \\ Email: *lauriann.young@uwimona.edu.jm
}

How to cite this paper: Young, L., Webber-Waugh, A. and Thaxter, K. (2021) DrugSeeking Behavior Is Significantly Attenuated in Nutritionally Supplemented Cocaine Withdrawn Sprague-Dawley Rats. Journal of Behavioral and Brain Science, 11, 143-156. https://doi.org/10.4236/jbbs.2021.117011

Received: May 18, 2021

Accepted: July 5, 2021

Published: July 8, 2021

Copyright $\odot 2021$ by author(s) and Scientific Research Publishing Inc. This work is licensed under the Creative Commons Attribution International License (CC BY 4.0).

http://creativecommons.org/licenses/by/4.0/

\begin{abstract}
Aim: The effect of patented nutritional supplementation on drug-seeking behavior in cocaine addicted rats during acute drug withdrawal was investigated using a biased Conditioned Place Preference (CPP) paradigm. Method: Twenty-four (24) male Sprague-Dawley rats with pre-conditioned preference for the black chamber of the CPP box were randomly divided into Cocaine $(\mathrm{COC})$ or Saline $(\mathrm{SAL})$ treated groups. Rats $(\mathrm{n}=12)$ treated with cocaine hydrochloride $20 \mathrm{mg} / \mathrm{kg} / \mathrm{ml}$, i.p. (COC group) were confined individually to the white chamber on days $1,3,5$ and 7 . On alternate days, they were given $1 \mathrm{ml}$ saline vehicle, i.p. and confined to the black chamber. Control rats (SAL group, $\mathrm{n}=12$ ) received only vehicle on all 8 days and were confined on alternate days to the white or black chamber. Positive place preference was confirmed for $\mathrm{COC}$ rats, which subsequently received 6 increasing daily doses of cocaine. CPP performances of both COC and SAL rats were recorded following an acute 3-day withdrawal period. All animals were then randomly assigned to rats fed either chow reconstituted with the nutritional supplement (COC-S and SAL-S) or standard rat chow (COC-N and SAL-N) for 8 weeks, followed by final CPP performances. Results: Following supplementation, COC-S rats made significantly less entries and time spent in the white chamber $(\mathrm{p}<0.05)$ compared with COC-N rats. COC-S rats exhibited significant place aversion to the white chamber similar to drug-naive animals; whereas COC-N continued to show positive place preference. Conclusion: Drug-seeking behavior that persisted during cocaine withdrawal was significantly diminished in the nutritionally supplemented.
\end{abstract}

\section{Keywords}

Cocaine, Conditioned Place Preference, Nutritional Supplement, Addiction, Drug-Seeking Behavior 


\section{Introduction}

Thirty-one million persons internationally suffer from substance use disorders (SUDs) with cocaine related SUDs being prevalent in the Caribbean, Latin America, North America and Western and Central Europe [1] [2]. Cocaine is a highly addictive central nervous system stimulant that can cause severe long-term effects on its users. Cocaine dependence or addiction is a public health problem. Recovery from the destructive factors inherent to cocaine addiction remains critically low not only because of limited health services, but also because of the unrelenting physiological addiction which must be overcome to cease drug-seeking and prevent recidivism.

Cocaine Use Disorders (CUDs) have been associated with alterations in the dorsolateral, prefrontal and dorsal anterior cingulate cortices [3]. Specifically, increased activity of glutamatergic pyramidal neurons in the medial Prefrontal Cortex (mPFC) that project to the Nucleus Accumbens (NAc) has been associated with cocaine-seeking behavior in rodent models of addiction [4]. These neurons also increase in synaptic plasticity following drug consumption [5]. This means that over time, drug-seeking behavior becomes a part of an increasingly complex arousal activity. Compulsive drug-seeking and craving, consumption and relapse are hallmark features of cocaine addiction, fueled by drug associated environmental stimuli that initiate cocaine associated memory [6]. The Conditioned Place Preference (CPP) paradigm is a well-established animal model utilized to study the role of context associations in reward-related behaviors, including both natural rewards and drugs of abuse [7]. This paradigm facilitates the creation of long-term reward related memory of the drug experience in its specific context and associated stimulus [8]. The expression of cocaine-associated memory using the CPP paradigm has also been linked to the activation of GABAergic neurons in the NAc [6].

Foods and supplements containing vitamins, minerals, fatty acids, carotenoids, polyphenols, bioactive peptides, probiotics, creatine and/or saponins as active ingredients have been observed as having significant therapeutic potential in treating drug addiction [9]. Anti-stress, anti-oxidant, anti-inflammatory, gut brain axis and neuro-protective pathways have all been proposed as possible mechanisms of action. Whilst the mechanism of action is yet to be elucidated, neuronal and cognitive protective roles have been hypothesized. This is further supported by research revealing B-vitamins, minerals, omega- 3 fatty acids and amino acids as being exceptionally deficient in mental disorder sufferers [10]. For example, it was reported that $75 \%$ of psychiatric patients presenting at a clinic had less than 4\% Eicosapentaenoic Acid (EPA) and Docosahexaenoic Acid (DHA) of total blood fatty acids and had improvement in psychiatric symptoms when treated with fish oil [11]. Fish oil supplementation was also reported to reduce stress increased peripheral ghrelin levels in rat serum with an associated antidepressant effect [12]. Ghrelin works via the mesolimbic dopaminergic pathway to potentiate rewarding behaviors in rodents, similar to the action of 
cocaine. Additionally, amino acid supplementation has been found to be critical for neurotransmitter synthesis, thus treatment with tyrosine was reported to improve dopamine and noradrenaline deficiencies [13]. Treatment strategies which therefore modulate these physiological activities may provide good therapeutic potential in drug addiction [14] [15] [16].

The unpleasant physical, emotional and mental symptoms associated with the sudden or acute withdrawal of cocaine result in changes in mood and appetite, often leading to recidivism and return to drug-seeking behavior. Furthermore, disordered and dysfunctional eating patterns pervade attempts at abstinence, potentially worsening the withdrawal experience of the addict [17]. Nutritional supplementation is therefore recommended early in treatment to supplement calories, regularize neurotransmitter regulation and restore of gut health [17] [18]. Therefore, the aim of this study was to investigate the effectiveness of a formulated nutritional supplement comprised of vitamins, minerals, amino and fatty acids on drug-seeking behavior of cocaine addicted Sprague-Dawley rats during drug withdrawal using a biased CPP paradigm.

\section{Materials and Method}

\subsection{Subjects}

Twenty-four (24) three (3) month old male Sprague-Dawley rats weighing approximately 250 - $350 \mathrm{~g}$, were obtained from the Animal House facility at The University of the West Indies (UWI) following institutional ethical approval of the protocol by the UHWI/FMS/UWI Ethics Committee. Animals were housed in pairs, in a normal 12:12 hour light: dark cycle and fed food and water ad libitum.

\subsection{The Behavioral Apparatus}

A three chambered CPP apparatus, measuring $75 \mathrm{~cm} \times 30 \mathrm{~cm} \times 25 \mathrm{~cm}$, as described in [17] was employed. The single black, white and grey chambers could each be isolated from the other by a sliding door on either side of the central grey corridor, which represented the starting chamber for exploration. A Perspex lid was used to cover the CPP box to facilitate viewing and video recording of the animal's exploratory behavior. The white chamber floor was covered with shiny transparent Perspex; while a wire mesh and saw-dust were placed on the floor of the black chamber, thus creating two contrasting chambers with distinct visual and tactile cues. The apparatus was thoroughly cleaned with a dilute solution of disinfectant following exploration by each rat.

\subsection{The Conditioned Place Preference (CPP) Paradigm}

Phase 1: Pre-conditioning, Selection and Group Assignments

Animals were allowed an initial twenty (20) minute exploration of the CPP apparatus, and the number of chamber entries and time spent in each chamber were recorded manually and via video recordings. An entry was recorded when 
all four paws of the rat were placed in a chamber. Animals displaying at least $80 \%$ preference for the black CPP chamber during pre-conditioning, assessed as a greater number of entries and time spent in the black chamber, were selected and randomly assigned to one of two treatment groups, COC (cocaine hydrochloride) or SAL ( $0.9 \%$ normal saline), each containing twelve (12) rats.

Phase 2: White Chamber Conditioning for Cocaine

Rats assigned to the cocaine treatment group (COC) received intra-peritoneal injections (i.p.) of $20 \mathrm{mg} / \mathrm{kg} / \mathrm{ml}$ of cocaine hydrochloride (Sigma, USA) dissolved in normal saline $(0.9 \%)$; while rats assigned to the SAL group received only vehicle ( $1 \mathrm{ml} 0.9 \%$ saline, i.p.). During the 8 -day conditioning period, group-assigned daily i.p. injections was followed immediately by individual confinement in the white, "non-preferred" or black "preferred" chamber of the CPP box for thirty (30) minutes. On days 1, 3, 5 and 7, COC rats received cocaine hydrochloride (Sigma, USA) while vehicle treated SAL rats received saline. Each rat in groups COC and SAL was then confined individually to the white "non-preferred" chamber. On the four alternate days (i.e., Days 2, 4, 6, 8), all animals received an injection of saline $(1 \mathrm{ml}$, i.p.) and were confined individually for 30 minutes in the black "preferred" chamber. Thus, COC rats were conditioned to pair cocaine with the white chamber and saline with the black chamber [9] [10]. On Day 9, rats were placed individually in the grey chamber, sliding doors then removed and rats allowed to explore the CPP box. The number of entries and time spent in the white chamber were recorded for 20 minutes and the percentage entries and time spent calculated from the total. Data obtained revealed that all animals assigned to the COC group exhibited a significant positive place preference drug-seeking behavior for the white chamber.

Phase 3: Cocaine Addiction and Drug Withdrawal

The addiction phase then ensued, with daily doses of cocaine hydrochloride $(20,20,25,25,30,30 \mathrm{mg} / \mathrm{kg} / \mathrm{ml}, i . p$.$) , increasing in concentration over a 6-day$ period. Group SAL rats received only vehicle (i.p.) over the same period and represented the non-drug controls for the addiction phase of the study. Rats were subsequently taken through a period of 3 days without drug treatment, vehicle or CPP exposure, and were fed standard rat chow with water ad libitum. On day 4 of drug withdrawal, each animal was individually placed in the grey start chamber and allowed a 20-minute period of exploration of the CPP box after the sliding doors were removed (Figure 1). The number of entries made into, and the time spent in each chamber, were recorded manually for all rats and the data compared with scores obtained from video recordings of rat's performance in the CPP paradigm.

Phase 4: Eight Weeks of Oral Nutritional Supplementation (S)

The orally administered, nutritional supplementation regimen used in this study consisted of three separate pharmaceutical grade components: 1) a megavitamin with higher levels of calcium and magnesium; 2) omega 3 and 6 fish oils; and 3) a supplement capsule consisting of basic amino acids with higher levels of 
Phase 1: CPP Pre-conditioning = Initial 20-minute

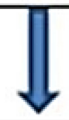

Rats $(n=24)$ exhibiting $\geq 80 \%$ black chamber preference

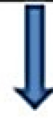

Phase 2: CPP Conditioning = 8-day i.p. drug treatment, CPP performance

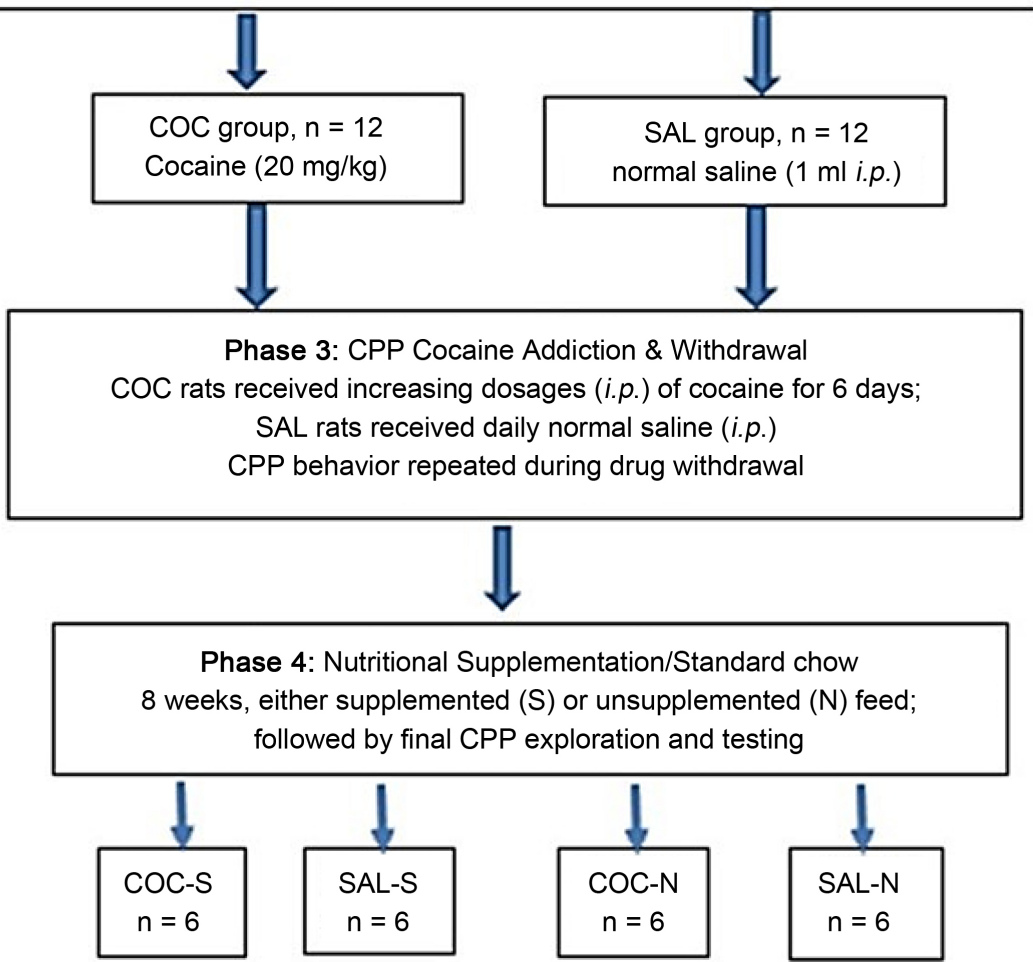

Figure 1. Flow diagram showing all phases of the experiment. Twenty-four (24) rats showing black-chamber preference were divided into two groups of 12 for treatment with cocaine $20 \mathrm{mg} / \mathrm{kg} / \mathrm{ml}$ (COC) or $0.9 \%$ normal saline (SAL). Six (6) rats from each treatment group were then given nutritional supplementation during withdrawal from cocaine. Performance in the CPP paradigm was assessed pre- and post-conditioning, during withdrawal and again following the supplementation or normal feeding period.

tyrosine (Manufacturer: All The Whey, Inc. Paoli PA, USA).

Nutritionally supplemented animals were proportionally fed a combination of the named component, at $0.123 \mathrm{~g} / \mathrm{day}, 0.123 \mathrm{~g} /$ day and $0.0408 \mathrm{~g} / \mathrm{day}$, respectively, over a period of 8 weeks. These doses were calculated based on a $350 \mathrm{~g}$ body weight rat, after proportions previously used in other studies for administration to a $75 \mathrm{~kg}$ man were scaled down [11]. Preparation of the nutritional supplement for oral treatment involved crushing and weighing the megavitamins; whereas, the fish oil tablets were opened and drained into a calibrated beaker and the relevant volumes removed. Supplement capsules were also opened, and the powder weighed for correct dosing. All animals were individually caged for the dura- 
tion of the supplementation period. The initial COC and SAL rat groups were further equally divided, assigned a numerical code and randomly assigned to either the supplemented diet or standard rat chow group, as indicated the four named groups below:

1) COC-S: Cocaine treated rats, fed with the nutritional supplement after the addiction phase.

2) COC-N: Cocaine treated rats, fed with standard rat chow throughout the experiment.

3) SAL-S: Saline treated rats, fed with the nutritional supplement after the addiction phase.

4) SAL-N: Saline treated rats, fed with standard rat chow throughout the experiment.

COC-S and SAL-S rats were individually housed in cages and received the nutritionally supplemented chow as first feed daily for the 8-week treatment period. Non-supplemented, standard rat chow was administered for the rest of the day, only after chow with supplement was fully consumed. The non-supplemented groups (i.e. COC-N and SAL-N) were administered food and water ad libitum throughout the day. Following this 8-week period, animals were randomly selected and individually placed in the CPP box and their performance in a final 20-minute exploration of the paradigm was assessed from video-recordings. Figure 1 is a graphical summary flow chart which explains the phases of the experiment. Data on time spent and entries in the drug-paired, white chamber were collated for the different groups after aligning scores obtained to the numerical code assigned to rats.

\section{Data Analysis}

Data on the mean percent time spent in (from a total time of 20 minutes) and entries (from the total arm entries of the CPP box during 20 minutes) into the white CPP chamber by each animal were analyzed using the SPSS-19 software. Mean scores for white and black chamber percent entries and time spent during the pre-conditioning phase, the withdrawal period and after the 8 weeks of supplemented feeding versus standard diet, were analyzed within groups using Student's T-test. Comparisons of the mean percent time and entries into both CPP chambers were also measured between groups, and analyzed using one-way ANOVA. Post-hoc analysis was performed using the Tukey test due to the distribution of the group means.

\section{Results}

1) Pre-conditioning exploratory behavior in the CPP paradigm

A significantly higher baseline mean percent entries into and time spent in the black chamber $(\mathrm{p}<0.05)$ were observed for all rat groups during the pre-conditioning phase.

2) Comparison of mean percent time spent in and percent entries into the 
white, drug-paired CPP chamber for groups COC-N and COC-S during preconditioning, cocaine withdrawal, and following 8 weeks of either nutritional supplementation or standard rat chow feeding

A summary of the CPP performance for all treatment groups in the white, non-preferred chamber that had been paired with cocaine is illustrated in Table 1 .

Cocaine withdrawn animals spent significantly more time in (COC-N, p = 0.015; COC-S, $\mathrm{p}=0.008$ ) and made more entries into (COC-N, $\mathrm{p}=0.009$; COC-S, $p=0.003)$ the drug-paired, white chamber during withdrawal when compared to their initial pre-conditioned performance. The non-supplemented group, COC-N, continued to spend more time in the drug-paired, white chamber after 8 weeks of standard rat chow consumption, and showed no significant place aversion to the white chamber throughout the cocaine withdrawal period. Following 8 weeks of nutritional supplementation, COC-S rats spent significantly less time in $(p=0.020)$ and made significantly fewer entries $(p=0.001)$ into the drug-paired, white chamber than they did during the acute drug withdrawal period (Figure 2 and Figure 3). There was no significant difference found between the initial pre-conditioned entries into the white chamber for supplemented cocaine-withdrawn rats (COC-S).

3) Comparison of percent entries into the white, drug-paired arm by COC and SAL groups during drug withdrawal, and after 8 weeks of nutritionally supplemented or standard rat chow consumption

ANOVA revealed that the cocaine treated groups, COC-N and COC-S, both made significantly higher percent entries into the white, drug-paired chamber $(\mathrm{p}=0.007)$ and $(\mathrm{p}=0.003)$ respectively, than did the vehicle treated SAL rats during the acute withdrawal phase of the experiment $(\mathrm{F}=32.64)$ (Figure 4). Group COC-N made significantly more entries into the white chamber compared with COC-S rats $(\mathrm{p}<0.001)$, as well as supplemented and non-supplemented drug-naïve rats, SAL-N and SAL-S $(\mathrm{p}<0.001)$. There was no significant difference found in white chamber entries among supplemented COC-S rats and their supplemented and un-supplemented vehicle controls, SAL-N and SAL-S.

Table 1. Summary of the Means \pm SEM for Time Spent in, and Entries into the Drug-paired, White CPP Chamber for the Treatment Groups [cocaine treated rats that did not receive supplementation (COC-N), cocaine treated rats that received nutritional supplementation (COC-S), saline treated rats that did not receive supplementation (SAL-N) and saline treated rats that received supplementation (SAL-S)] in the Three Phases of the Study Design.

\begin{tabular}{|c|c|c|c|c|c|c|c|c|}
\hline \multirow[b]{2}{*}{ Treatment Groups } & \multicolumn{4}{|c|}{$\begin{array}{c}\text { Mean Percent Time Spent } \pm \text { SEM } \\
\text { in the CPP White Chamber }\end{array}$} & \multicolumn{4}{|c|}{$\begin{array}{c}\text { Mean Percent Entries } \pm \text { SEM into } \\
\text { the CPP White Chamber }\end{array}$} \\
\hline & COC-N & COC-S & SAL-N & SAL-S & $\mathrm{COC}-\mathrm{N}$ & COC-S & SAL-N & SAL-S \\
\hline Pre-Conditioning Phase & $1.35 \pm 0.57$ & $1.03 \pm 0.49$ & $1.13 \pm 0.53$ & $1.22 \pm 0.46$ & $13.6 \pm 3.27$ & $10.52 \pm 1.85$ & $13.5 \pm 0.77$ & $14.67 \pm 1.54$ \\
\hline Cocaine Withdrawal Phase & $20.89 \pm 5.21$ & $22.5 \pm 5.25$ & $1.07 \pm 0.30$ & $0.97 \pm 0.48$ & $26.00 \pm 2.93$ & $27.29 \pm 1.75$ & $11.73 \pm 0.55$ & $8.07 \pm 0.15$ \\
\hline $\begin{array}{l}8 \text { weeks feeding with } \\
\text { standard or suppl. rat chow } \\
\text { after acute drug withdrawal }\end{array}$ & $17.55 \pm 4.74$ & $13.10 \pm 6.25$ & $1.08 \pm 0.36$ & $1.58 \pm 0.65$ & $25.65 \pm 2.42$ & $9.40 \pm 2.88$ & $9.81 \pm 0.65$ & $5.85 \pm 1.69$ \\
\hline
\end{tabular}




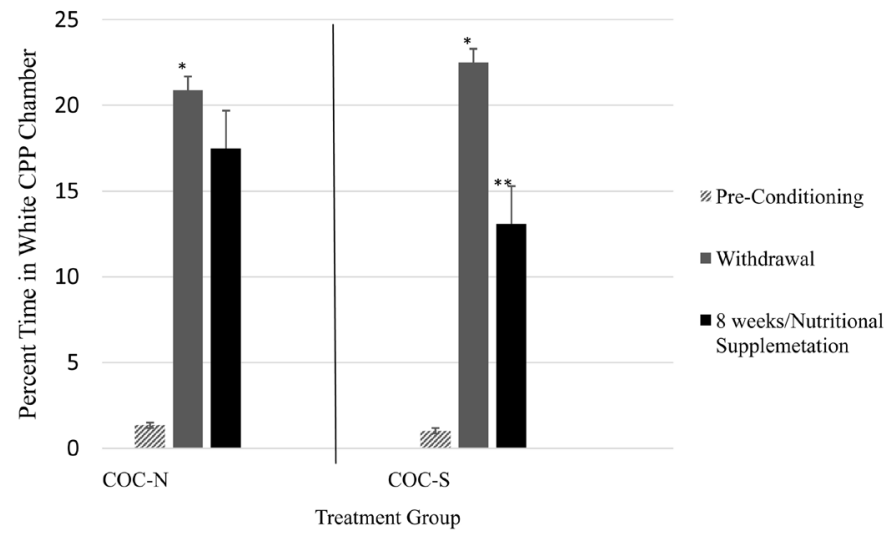

Figure 2. A comparison of the mean percent time in the white, drug-paired CPP chamber for cocaine-addicted rats following 3 days of drug withdrawal and 8 weeks of nutritionally supplemented (COC-S group, $\mathrm{n}=6$ ) and non-supplemented feeding with standard rat chow (COC-N group, $n=6$ ). There was a significant increase in white chamber time by both drug treated groups compared to their initial pre-conditioning time, and after acute drug withdrawal. COC- $\mathrm{N}$ rats spent significantly more time in the white chamber after 8 weeks of standard rat chow; whereas COC-S rats spent significantly less time in the white chamber after 8 weeks of nutritional supplementation. There was no significant difference from pre-conditioning percent white chamber time for nutritional supplemented cocaine-withdrawn rats (COC-S) ${ }^{*} \mathrm{p}<0.05$ compared to pre-conditioning time; ${ }^{* *} \mathrm{p}<0.05$ compared to time spent after 3 days of drug withdrawal. Values are expressed as the mean percent \pm S.E.M.

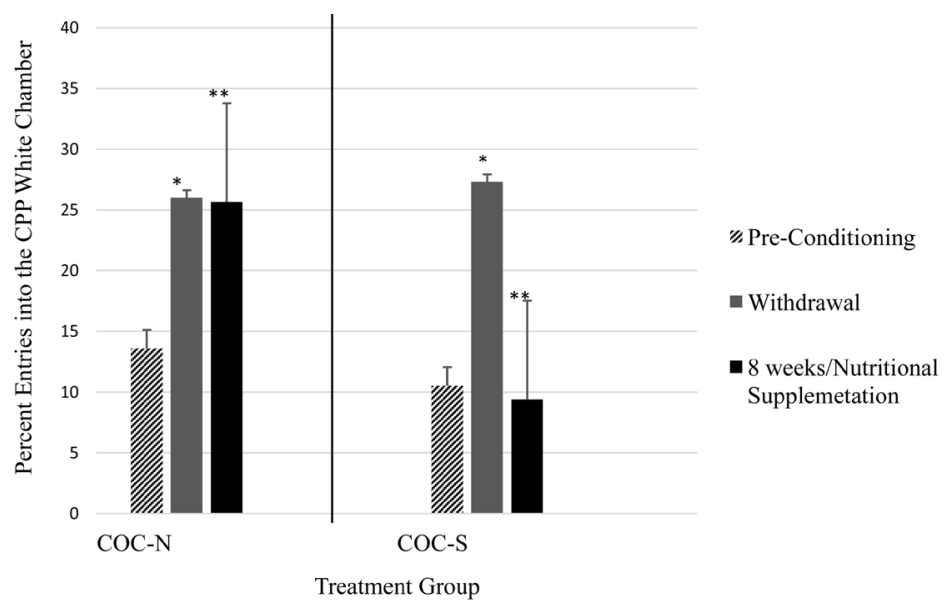

Figure 3. A comparison of the mean percent entries into the white, drug-paired CPP chamber for cocaine-addicted rats following 3 days of drug withdrawal and 8 weeks of nutritionally supplemented (COC-S group, $\mathrm{n}=6$ ) and non-supplemented feeding with standard rat chow (COC-N group, $\mathrm{n}=6$ ). There was a significant increase in white chamber entries by both cocaine treated rats groups compared to the initial pre-condition time and after acute drug withdrawal. Non-supplemented cocaine-withdrawn rats (COC-N) continued to make significantly more entries into the white chamber after 8 weeks of standard rat chow; whereas supplemented cocaine-withdrawn rats (COC-S) made significantly less entries into the white chamber after 8 weeks of nutritional supplementation. COC-S rats showed no significant difference in percent white chamber entries from their initial pre-condition entries. ${ }^{*} \mathrm{p}<0.05$ compared to pre-conditioning entries; ${ }^{* *} \mathrm{p}<0.05$ compared to entries after 3 days of drug withdrawal. Values are expressed as the mean \pm S.E.M. 


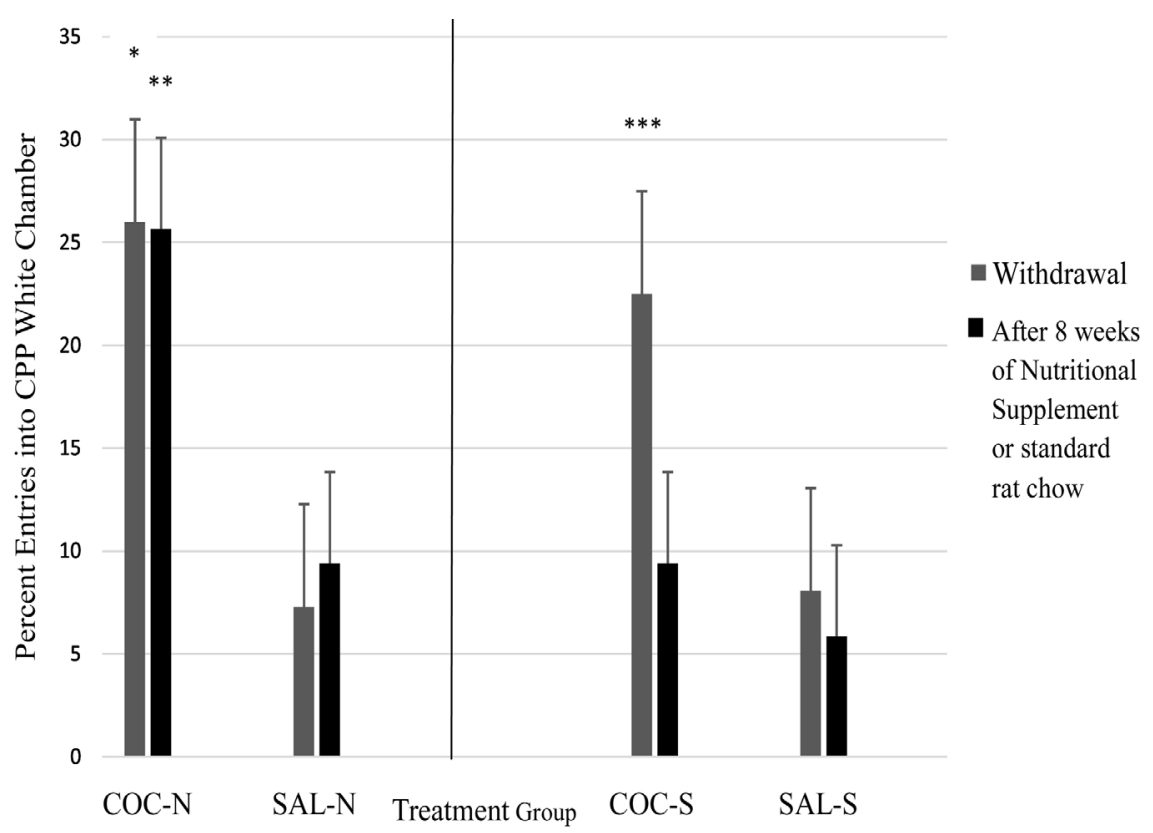

Figure 4. A comparison of the mean percent entries into the white, drug-paired CPP chamber for all COC and SAL groups during withdrawal and after 8 weeks of nutritional supplementation or standard chow feeding. Animals in group COC-N made significantly more entries into the white CPP chamber during withdrawal than did animals in group SAL-N. COC-N rats continued to make significantly more entries than SAL-N into the white chamber after 8 weeks of standard chow consumption. During withdrawal, COC-S made significantly more entries into the white, drug-paired chamber than did animals in group SAL-S. After 8 weeks of nutritional supplementation, group COC-S showed no significant difference from group SAL-S in mean percent white chamber entries. $\mathrm{N}=6$ for both groups. ${ }^{*} \mathrm{p}<0.05$ compared to SAL-N during withdrawal; ${ }^{* *} \mathrm{p}<0.05$ compared to SAL-N after 8 weeks normal rat chow; ${ }^{* *} \mathrm{p}<0.05$ compared to SAL-S during withdrawal. All values are expressed as mean percent \pm S.E.M.

4) Comparison of percent time spent in the white and black CPP chambers by COC and SAL groups during pre-conditioning, post drug, and after 8 weeks of supplemented or standard rat chow consumption

Both COC-N and COC-S groups spent significantly more time in the drug-paired white CPP chamber during withdrawal $(\mathrm{p}<0.022)$ than their saline treated counterparts, SAL-N and SAL-S, according to ANOVA analysis ( $\mathrm{F}=$ 32.64). COC groups also made significantly more entries into the white chamber during withdrawal, than did SAL groups ( $\mathrm{p}<0.008)$.

Time spent in the naturally preferred black chamber was significantly lower for both COC-N and COC-S groups during withdrawal $(\mathrm{p}<0.001)$ than displayed by drug-naïve SAL-N and SAL-S groups, indicating place preference for the drug-paired chamber. However, after 8 weeks, nutritionally supplemented COC-S rats displayed no significant difference in black chamber time when compared to the non-drug exposed, SAL groups $(\mathrm{p}<0.001)$. In contrast, un-supplemented, cocaine-withdrawn COC-N rats displayed place aversion to the black chamber $(\mathrm{F}=32.64)$ (Figure 5). 


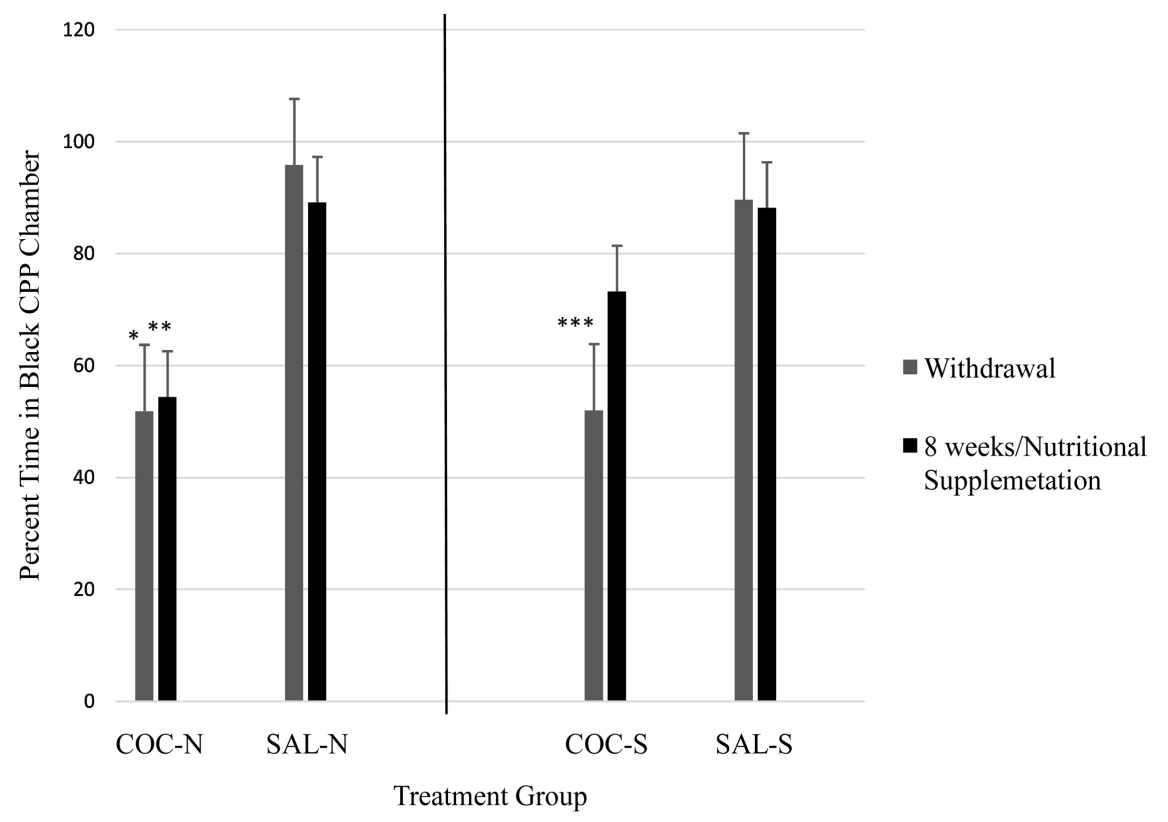

Figure 5. A comparison of the mean percent time spent in the black, preferred CPP chamber for all COC and SAL groups during drug withdrawal and after 8 weeks of nutritional supplementation or standard chow feeding. Animals in groups COC-N and COC-S spent significantly less time in the black CPP chamber during withdrawal than did animals in groups SAL-N and SAL-S. COC-N rats continued to spend significantly less time than SAL-N in the black chamber after 8 weeks of standard chow consumption. After 8 weeks of nutritional supplementation, group COC-S showed no significant difference from group SAL-S in mean percent time in the black chamber. $\mathrm{n}=6$ for both groups. ${ }^{*} \mathrm{p}$ $<0.05$ compared to SAL-N during withdrawal; ${ }^{* *} \mathrm{p}<0.05$ compared to SAL-N after 8 weeks of normal rat chow; ${ }^{* *} \mathrm{p}<0.05$ compared to SAL-S during withdrawal. All values are expressed as mean percent \pm S.E.M.

\section{Discussion}

The results of this study revealed that consumption of the patented nutritional supplement reversed cocaine-induced positive place preference in cocaine addicted rats. We demonstrated significant decreases in the percent time spent in and the number of entries into the drug-paired, white chamber of the CPP paradigm, when cocaine withdrawn rats were nutritionally supplemented for 8 weeks. Non-supplemented Cocaine-withdrawn rats (COC-N) continued to exhibit significant place preference for the drug-paired chamber during the drug withdrawal phase and subsequently, following 8 weeks of standard chow consumption, when compared with their Saline Treated Counterparts (SAL-N).

The CPP paradigm is a well-established model to evaluate motivational effects of drugs [19] and factors that potentially affect relapse to drug abuse such as non-drug treatment strategies [20]. The initial baseline preference for the black CPP chamber or place aversion for the white chamber exhibited by all rats in the study as well as the significant place preference or drug-seeking behavior by cocaine treated animals for the white, non-preferred, drug-paired chamber validated the design of the paradigm adopted. 
Examination of the performance of each treatment group in this experiment on a timeline from pre-conditioning to post 8-week supplementation vs standard chow provided a reinforcement of the transition of the cocaine addicted, withdrawn animals from heightened drug-seeking to a convincing return to behavior recorded prior to drug exposure, after supplementation. These findings provide compelling evidence that successful management of cocaine withdrawal is heavily dependent on the manipulation of diet during recovery.

Cocaine use has been associated with ischaemic colitis so frequently that it is recommended [21] that every young person diagnosed with this disorder be investigated for cocaine misuse. Cocaine is a potent sympathomimetic mesenteric vasoconstrictor that produces severe small or large intestinal ischemia, often requiring laparotomy for gangrenous bowel. Maldigestion (defined as the defective intraluminal hydrolysis of nutrients) or malabsorption (defined as defective mucosal absorption) are both functional results of bowel ischaemia [22]. When the ischaemia affects the epithelial brush border of the small intestine, amino acid absorption is impaired. Cocaine deranges the dopaminergic system, producing cravings after the "high" that can be modulated by antagonizing dopamine D1, D2 and D3 receptor subtypes [23]. Symptoms of cocaine seeking have been attributed to dopamine depletion [24]. It is proposed that the megavitamin, fish oil and amino acid capsules of the patented nutritional supplement used in this study potentiated improved gastrointestinal function during drug withdrawal, improved amino acid and general nutrient absorption, and potentiated the recreation of the neurotransmitter balance that accompanies "normal" behavior.

Termination of an addictive drug often causes the addict to experience a combination of physical and emotional symptoms of discomfort, agitation, sleeplessness, nausea and vomiting, intense drug craving, anxiety, dysphoria, extreme fatigue, and apathy among others as the body tries to adjust to a state of non-dependence. These effects are often intense in the acute phase of drug withdrawal, which in turn prolongs and sustains the drug addiction, leading inevitably to relapse to uncontrolled drug consumption or recidivism.

The results of this study provide further evidence that vitamin, mineral and amino acid therapy may reduce withdrawal symptoms in addicts using drugs such as cocaine and amphetamine, thus decreasing recidivism with better rehabilitation. A few non-drug treatment strategies have also been shown to modify gut microbiome which provides a possible mechanism by which such strategies are successful. For example, dietary supplementation with omega-3 polyunsaturated fatty acids has been used to reduce opioid seeking behavior and stabilize the gut microbiome [2]. Additionally, high fat diets have been used generally to modify drug-seeking behavior and alter gut microbiome [1]. Such evidence may provide a possible explanation for the success provided by the nutritional supplement used in our investigation which is rich in omega 3 and other fatty acids. Other research indicates that $\beta$-Lactam antibiotics and sodium butyrate may also 
remove drug-seeking by altering gut microbiome [25].

\section{Acknowledgements}

The team wishes to thank UWI Mona Campus Office of Graduate Studies and Research for providing funding for the study. Many thanks also to Dr. A. Salako-Akande for making available her proprietary formulation of the nutritional supplement that was used in this research.

\section{Conflicts of Interest}

There is no conflict of interest.

\section{References}

[1] Hakimian, J.K., Dong, T.S., Barahona, J.A., Lagishetty, V., Tiwari, S., Azani, D., Barrera, M., Lee, S., Severino, A.L., Mittal, N., Cahill, C.M., Jacobs, J.P. and Walwyn, W.M. (2019) Dietary Supplementation with Omega-3 Polyunsaturated Fatty Acids Reduces Opioid-Seeking Behaviors and Alters the Gut Microbiome. $\mathrm{Nu}$ trients, 11, 1900. https://doi.org/10.3390/nu11081900

[2] Aguilar, M.A., Rodríguez-Arias, M. and Miñarro, J. (2009) Neurobiological Mechanisms of the Reinstatement of Drug-Conditioned Place Preference. Brain Research Reviews, 59, 253-277. https://doi.org/10.1016/j.brainresrev.2008.08.002

[3] Martinez, D., Urban, D., Grassetti, A., Chang, D., Mei-Chen H., Zangen, A., Levin, F.R., Foltin, R. and Nunes, E.V. (2018) Transcranial Magnetic Stimulation of Medial Prefrontal and Cingulate Cortices Reduces Cocaine Self-Administration: A Pilot Study. Frontiers in Psychiatry, 9, 80. https://doi.org/10.3389/fpsyt.2018.00080

[4] Slaker, M.L., Jorgensen, E.T., Hegarty, D.M., Liu, X., Kong, Y., Zhang, F., Linhardt, R.J., Brown, T.E., Aicher, S.A. and Sorg, B.A. (2018) Cocaine Exposure Modulates Perineuronal Nets and Synaptic Excitability of Fast-Spiking Interneurons in the Medial Prefrontal Cortex. eNeuro, 5, e0221-18. https://doi.org/10.1523/ENEURO.0221-18.2018

[5] Otis, J.M., Fitzgerald, M.K., Yousuf, H., Burkard, J.L., Drake, M. and Mueller, D. (2018) Prefrontal Neuronal Excitability Maintains Cocaine-Associated Memory during Retrieval. Frontiers in Behavioral Neuroscience, 12, 119.

https://doi.org/10.3389/fnbeh.2018.00119

[6] Zhang, T., Deyama, S., Domoto, M., Wada, S., Yanagida, J., Sasase, H., Hinoi, E., Nishitani, N., Nagayasu, K., Kaneko, S. and Kaneda, K. (2018) Activation of GABAergic Neurons in the Nucleus Accumbens Mediates the Expression of Cocaine-Associated Memory. Biological and Pharmaceutical Bulletin, 41, 1084-1088. https://doi.org/10.1248/bpb.b18-00221

[7] McKendrick, G. and Graziane, N.M. (2020) Drug-Induced Conditioned Place Preference and Its Practical Use in Substance Use Disorder Research. Frontiers in Behavioral Neuroscience, 14, Article ID: 582147. https://doi.org/10.3389/fnbeh.2020.582147

[8] Salti, A., Apostolova, G., Kummer, K.K., Lemos, C. Dechant, G. and El Rawas, R. (2018) Cocaine Paired Environment Increases SATB2 Levels in the Rat Paraventricular Thalamus. Frontiers in Behavioral Neuroscience, 12, 224. https://doi.org/10.3389/fnbeh.2018.00224

[9] Frutos, M.J., Valero-Cases, E. and Rincon-Frutos, L. (2019) Food Components with Potential to Be Used in the Therapeutic Approach of Mental Diseases. Current 
Pharmaceutical Biotechnology, 20, 100-113. https://doi.org/10.2174/1389201019666180925120657

[10] Lakhan, S.E. and Vieira, K.F. (2008) Nutritional Therapies for Mental Disorder. Nutrition Journal, 7, Article No. 2. https://doi.org/10.1186/1475-2891-7-2

[11] Messamore, E. and McNamara, R.K. (2016) Detection and Treatment of Omega-3 Fatty Acid Deficiency in Psychiatric Practice: Rationale and Implementation. Lipids in Health and Disease, 15, Article No. 25. https://doi.org/10.1186/s12944-016-0196-5

[12] Tang, M., Jiang, P., Li, H., Liu, Y., Cai, H., Dang, R., Zhu, W. and Cao, L. (2015) Fish Oil Supplementation Alleviates Depressant-Like Behaviors and Modulates Lipid Profiles in Rats Exposed to Chronic Unpredictable Mild Stress. BMC Complementary and Alternative Medicine, 15, Article No. 239. https://doi.org/10.1186/s12906-015-0778-1

[13] McLean, A., Rubinsztein, J.S., Robbins, T.W., Sahakian, B.J., McLean, A., Rubinsztein, J.S., Robbins, T.W. and Sahakian, B.J. (2004) The Effects of Tyrosine Depletion in Normal Healthy Volunteers: Implications for Unipolar Depression. Psychopharmacology, 171, 286-297. https://doi.org/10.1007/s00213-003-1586-8

[14] Revitsky, A.R. and Klein, L.C. (2013) Role of Ghrelin in Drug Abuse and Reward-Relevant Behaviors: A Burgeoning Field and Gaps in the Literature. Current Drug Abuse Reviews, 6, 231-244. https://doi.org/10.2174/1874473707666140205200532

[15] Huang, H.-J., Zhu, X.-C., Han, Q.-Q., Wang, Y.-L., Yue, N., Wang, J., Yu, R., Li, B., Wu, G.-C., Liu, Q. and Yu, J. (2017) Ghrelin Alleviates Anxiety- and Depression-Like Behaviors Induced by Chronic Unpredictable Mild Stress in Rodents. Behavioral Brain Research, 326, 33-43. https://doi.org/10.1016/j.bbr.2017.02.040

[16] Morris, L.S., Voon, V. and Leggio, L. (2018) Stress Motivation, and the Gut-Brain Axis: A Focus on the Ghrelin System and Alcohol Use Disorder. Alcoholism Clinical and Experimental Research, 42, 1378-1389. https://doi.org/10.1111/acer.13781

[17] Webber-Waugh, A., Thaxter-Nesbeth, K., Anderson-Johnson, P., Salako-Akande, A., Asemota, H. and Young, L. (2017) Drug Seeking Behavior of Amphetamine Addicted Sprague-Dawley Rats Is Eliminated after Nutritional Supplementation. Journal of Behavioral and Brain Science, 7, 585-597. https://doi.org/10.4236/jbbs.2017.712041

[18] Wiss, D.A. and Waterhouse, T.S. (2014) Nutrition Therapy for Eating Disorders, Substance Use Disorders, and Addictions. In: Brewerton, T. and Baker, D.A., Eds., Eating Disorders, Addictions and Substance Use Disorders, Springer, Berlin, 509-532. https://doi.org/10.1007/978-3-642-45378-6 23

[19] Tzschentke, T.M. (2007) Review on CPP: Measuring Reward with the Conditioned Place Preference (CPP) Paradigm: Update of the Last Decade. Addiction Biology, 12, 227-462. https://doi.org/10.1111/j.1369-1600.2007.00070.x

[20] Blanco-Gandia, M.C., Aguilar, M.A., Minarro, J. and Rodriguez-Arias, M. (2018) Reinstatement of Drug-Seeking in Mice Using the Conditioned Place Preference Paradigm. Journal of Visualized Experiments, 136, e56983. https://doi.org/10.3791/56983

[21] Parfitt, J.R. and Driman, D.K. (2007) Pathological Effects of Drugs on the Gastrointestinal Tract: A Review. Human Pathology, 38, 527-536.

https://doi.org/10.1016/j.humpath.2007.01.014

[22] Montoro-Huguet, M.A., Belloc, B. and Domínguez-Cajal, M. (2021) Small and Large Intestine (I): Malabsorption of Nutrients. Nutrients, 13, 1254. 
https://doi.org/10.3390/nu13041254

[23] Koob, G.F., Sanna, P.P. and Bloom, F.E. (1998) Neuroscience of Addiction. Neuron, 21, 467-476. https://doi.org/10.1016/S0896-6273(00)80557-7

[24] Dackis, C.A., Gold, M.S. and Sweeney, D.R. (1987) The Physiology of Cocaine Craving and Crashing. Archives of General Psychiatry, 44, 298-299. https://doi.org/10.1001/archpsyc.1987.01800150122018

[25] Angoa-Pérez, M. and Kuhn, D.M. (2021) Evidence for Modulation of Substance Use Disorders by the Gut Microbiome: Hidden in Plain Sight. Pharmacological Reviews, 73, 571-596. https://doi.org/10.1124/pharmrev.120.000144 